\title{
A comparison of statistically optimized near field acoustic holography using single layer pressure velocity measurements and using double layer pressure measurements
}

\author{
Jacobsen, Finn; Chen, Xinyi; Jaud, Virginie
}

Published in:

Journal of the Acoustical Society of America

Link to article, DOI:

$10.1121 / 1.2875308$

Publication date:

2008

Document Version

Publisher's PDF, also known as Version of record

Link back to DTU Orbit

Citation (APA):

Jacobsen, F., Chen, X., \& Jaud, V. (2008). A comparison of statistically optimized near field acoustic holography using single layer pressure velocity measurements and using double layer pressure measurements. Journal of the Acoustical Society of America, 123(4), 1842-1845. https://doi.org/10.1121/1.2875308

\section{General rights}

Copyright and moral rights for the publications made accessible in the public portal are retained by the authors and/or other copyright owners and it is a condition of accessing publications that users recognise and abide by the legal requirements associated with these rights.

- Users may download and print one copy of any publication from the public portal for the purpose of private study or research.

- You may not further distribute the material or use it for any profit-making activity or commercial gain

- You may freely distribute the URL identifying the publication in the public portal 


\title{
A comparison of statistically optimized near field acoustic holography using single layer pressure-velocity measurements and using double layer pressure measurements $(L)^{\text {a) }}$
}

\author{
Finn Jacobsen ${ }^{\text {b) }}$ and Xinyi Chen ${ }^{\text {c) }}$ \\ Acoustic Technology, Ørsted·DTU, Technical University of Denmark, Building 352, Ørsteds Plads, \\ DK-2800 Kgs. Lyngby, Denmark \\ Virginie Jaud ${ }^{\text {d) }}$ \\ Laboratoire E3I2-EA3876, École Nationale Supérieure d'Ingénieurs des Études des Techniques \\ d'Armement, 2 rue François Verny, F-29806 Brest Cedex 9, France
}

(Received 23 January 2008; accepted 24 January 2008)

\begin{abstract}
Statistically optimized near field acoustic holography (SONAH) is usually based on the assumption that all sources are on one side of the measurement plane whereas the other side is source free. An extension of the SONAH procedure based on measurement with an array of pressure-velocity probes has recently been suggested. An alternative method uses a double layer array of pressure transducers. Both methods make it possible to distinguish between sources on the two sides of the array and thus suppress the influence of extraneous noise and reflections coming from the "wrong" side. This letter compares the two methods. (C) 2008 Acoustical Society of America.
\end{abstract}

[DOI: 10.1121/1.2875308]

PACS number(s): 43.60.Sx, 43.60.Pt, 43.20.Rz [EJS]

Pages: $1842-1845$

\section{INTRODUCTION}

Near field acoustic holography (NAH) is used for experimental analysis of sound fields near sources. ${ }^{1}$ Statistically optimized near field acoustic holography (SONAH) is a variant of NAH developed by Steiner and Hald in order to overcome the truncation errors associated with the spatial Fourier transform used in conventional NAH. ${ }^{2,3}$

$\mathrm{NAH}$ and SONAH are usually based on measurement of the sound pressure. However, it has recently been demonstrated that NAH based on measurement of both the pressure and the normal component of the particle velocity in general gives more accurate sound field reconstructions than NAH based exclusively on pressure measurements. ${ }^{4}$ The performance of SONAH is also improved if it is based on measurements of both quantities. ${ }^{5}$ An additional advantage of the pressure-velocity technique is that one can combine pressure- and velocity-based predictions, which makes it possible to distinguish between sound coming from the two sides of the measurement plane in the same way as one can do with a double layer array of pressure transducers. ${ }^{6,7}$ This makes it possible to distinguish, e.g., between incident and reflected waves, which can be very useful when measurements take place under nonideal conditions. However, it is far more difficult to calibrate particle velocity transducers than condenser microphones. ${ }^{8}$ Thus, the purpose of this letter

\footnotetext{
Portions of this work were presented in "Statistically optimized near field acoustic holography with pressure-velocity probes and with a double layer array," Proceedings of Inter-Noise 2007, Istanbul, Turkey, August 2007.

b) Author to whom correspondence should be addressed. Electronic mail: fja@oersted.dtu.dk

${ }^{c}$ Electronic mail: xinyi.cc@gmail.com

${ }^{\mathrm{d})}$ Electronic mail: virginie.jaud@ensieta.fr
}

is to compare SONAH based on pressure and velocity measurements in a single layer with SONAH based on pressure measurements in a double layer.

\section{OUTLINE OF SONAH THEORY}

The theory of SONAH has been presented, e.g., in Refs. 2 and 3; therefore the description given in what follows is very brief.

\section{A. SONAH based on measurement of the sound pressure}

The conventional SONAH procedure assumes that all sources are on one side of the measurement plane and expresses the pressures in the prediction plane as a weighted sum of pressures in the measurement plane,

$$
\mathbf{p}^{T}(\mathbf{r})=\mathbf{p}^{T}\left(\mathbf{r}_{h}\right)\left(\mathbf{A}^{H} \mathbf{A}+\theta^{2} \mathbf{I}\right)^{-1} \mathbf{A}^{H} \boldsymbol{\alpha}(\mathbf{r}),
$$

where $\mathbf{p}^{T}(\mathbf{r})$ is a transposed column vector with $N$ pressures in the prediction plane, $\mathbf{p}^{T}\left(\mathbf{r}_{h}\right)$ is a similar vector with $N$ pressures in the measurement plane, $\mathbf{A}^{H} \mathbf{A}$ is a matrix that depends on the $N$ positions in the measurement plane, $\mathbf{A}^{H} \boldsymbol{\alpha}$ is a matrix that depends on the $N$ positions in the prediction plane and the $N$ positions in the measurement plane, $\mathbf{I}$ is the identity matrix, and $\theta$ is a regularization parameter. ${ }^{3}$ All matrices are $N$ by $N$. The transfer matrix represents the regularized optimal least-squares solution to the overdetermined problem posed by requiring that $M$ propagating and evanescent elementary waves $(M>N)$, all having a pressure amplitude of unity in the virtual source plane, satisfy Eq. (1). In the limit of $M \rightarrow \infty$ all matrix elements become integrals that can be evaluated partly analytically and partly numerically. ${ }^{3}$ 
The normal component of the particle velocity in the prediction plane is obtained from a similar transfer matrix where $\mathbf{A}^{H} \boldsymbol{\alpha}$ is replaced by

$$
\mathbf{A}^{H} \boldsymbol{\beta}(\mathbf{r})=\frac{-1}{\mathrm{j} \omega \rho} \frac{\partial \mathbf{A}^{H} \boldsymbol{\alpha}(\mathbf{r})}{\partial z} .
$$

\section{B. SONAH based on measurement of the particle velocity}

The normal component of the particle velocity in the prediction plane can also be determined from the normal component of the particle velocity in the measurement plane using the same transfer matrix as in Eq. (1). ${ }^{5}$ The corresponding pressure can be determined from a similar transfer matrix where $\mathbf{A}^{H} \boldsymbol{\alpha}$ is replaced by

$$
\mathbf{A}^{H} \boldsymbol{\gamma}(\mathbf{r})=-\mathrm{j} \omega \rho \int \mathbf{A}^{H} \boldsymbol{\alpha}(\mathbf{r}) d z .
$$

The two transfer matrices for particle velocity-based SONAH are determined from an infinite set of propagating and evanescent elementary waves with a particle velocity amplitude of unity in the virtual source plane, unlike the two transfer matrices for pressure-based SONAH. Surprisingly, the weighting of the elementary waves has been found to have almost no influence on the results. ${ }^{9}$

\section{SONAH based on measurement of the pressure and the particle velocity}

One cannot distinguish between wave components coming from the two sides of the measurement array from measurements of the pressure or from measurements of the particle velocity; hence, the assumption of the region opposite to the source side of the measurement plane being "source free." However, the fact that the particle velocity is a vector component, unlike the pressure, makes it possible to separate contributions from two sides of the measurement array from each other when both quantities are available. ${ }^{5}$ Thus the sound pressure in the primary prediction plane generated by the primary source can be estimated as the average of a pressure- and a particle velocity-based estimate, and the sound pressure in the secondary prediction plane generated by a source on the other side of the measurement plane can be estimated as half the difference between a pressure- and a particle velocity-based estimate. The same holds for the particle velocity. In what follows this method of combining two estimates based on pressure and particle velocity measurements is referred to as "the $p-u$ method."

Note that the prediction plane for estimates based on subtracting pressure- and particle velocity-based predictions, here referred to as the secondary prediction plane, is the symmetrically placed image of the prediction plane for estimates based on averaging pressure- and particle velocitybased predictions, here referred to as the primary prediction plane. The reason for this is that the elementary waves used in determining the transfer matrices appear to be coming from the image of the virtual source plane.

\section{SONAH based on measurement of the pressure in two parallel planes}

An alternative to the $p-u$ method is based on measurement of the sound pressure in two parallel planes. The double layer technique, originally proposed by Tamura as an extension of $\mathrm{NAH}^{6}$ also works with SONAH. ${ }^{7}$ The sound pressure in the prediction plane is expressed as a weighted sum of sound pressures measured in the two planes $a$ and $b$, here assumed to be parallel,

$$
\begin{aligned}
\mathbf{p}^{T}(\mathbf{r})= & \left(\begin{array}{l}
\mathbf{p}\left(\mathbf{r}_{h}^{a}\right) \\
\mathbf{p}\left(\mathbf{r}_{h}^{b}\right)
\end{array}\right)^{T}\left(\left(\begin{array}{cc}
\mathbf{G}_{a a} & \mathbf{G}_{a b} \\
\mathbf{G}_{a b}^{H} & \mathbf{G}_{a a}
\end{array}\right)^{H}\left(\begin{array}{cc}
\mathbf{G}_{a a} & \mathbf{G}_{a b} \\
\mathbf{G}_{a b}^{H} & \mathbf{G}_{a a}
\end{array}\right)+\theta^{2} \mathbf{I}\right)^{-1} \\
& \times\left(\begin{array}{l}
\mathbf{h}_{a}(\mathbf{r}) \\
\mathbf{h}_{b}(\mathbf{r})
\end{array}\right)
\end{aligned}
$$

where $\mathbf{p}\left(\mathbf{r}_{h}^{a}\right)$ and $\mathbf{p}\left(\mathbf{r}_{h}^{b}\right)$ are column vectors with $N$ pressures in the two measurement planes, $\mathbf{G}_{a a}$ and $\mathbf{G}_{a b}$ are $N$ by $N$ matrices that depend on the $N$ positions in measurement plane $a$ and in both measurement planes, respectively, $\mathbf{I}$ is the identity matrix $(2 N$ by $2 N)$, and $\mathbf{h}_{a}$ and $\mathbf{h}_{b}$ are $N$ by $N$ matrices that depend both on the $N$ positions in the prediction plane and the $N$ positions in measurement plane $a$ and $b$, respectively. As noted earlier, the transfer matrix represents the regularized optimal least-squares solution to an overdetermined problem posed by requiring that a large number of propagating and evanescent elementary waves satisfy Eq. (4). However, now there are two virtual source planes placed symmetrically on either side of the two measurement planes, and two corresponding sets of elementary propagating and evanescent waves.

The normal component of the particle velocity can be determined from a similar transfer matrix where $\mathbf{h}_{a}$ and $\mathbf{h}_{b}$ are replaced by

$$
\left(\begin{array}{l}
\mathbf{h}_{a}^{(u)}(\mathbf{r}) \\
\mathbf{h}_{b}^{(u)}(\mathbf{r})
\end{array}\right)=\frac{-1}{\mathrm{j} \omega \rho} \frac{\partial}{\partial z}\left(\begin{array}{l}
\mathbf{h}_{a}(\mathbf{r}) \\
\mathbf{h}_{b}(\mathbf{r})
\end{array}\right) .
$$

In what follows the method based on measurement of the pressure in a double layer is referred to as "the DLA method."

\section{A SIMULATION STUDY}

A study with simulated measurements has been carried out. The $p-u$ method is compared with the DLA method for realistic, complicated sources. Regularization is done without human intervention using the generalized cross validation method. ${ }^{10}$

The measurement plane for the $p-u$ method is placed midway between the two source regions at $z=0$, and the two measurement planes for the DLA method are placed symmetrically at $z= \pm 1.5 \mathrm{~cm}$. All measurement arrays have dimensions of $21 \times 21 \mathrm{~cm}$ with $8 \times 8$ transducer positions. The prediction planes have the same dimensions. The two prediction planes of the $p-u$ method are placed symmetrically at $z= \pm 4.5 \mathrm{~cm}$, and the same prediction planes have been chosen for the DLA method. The virtual source plane for the $p-u$ method is at $z=-7.5 \mathrm{~cm}$, and the virtual source planes for the DLA method are at $z= \pm 7.5 \mathrm{~cm}$. In the primary prediction plane the sound field that would be generated by source re- 
gion 1 in the absence of the disturbance from source region 2 is predicted; and in the secondary prediction plane on the other side of the double layer array the sound field that would be generated by source region 2 in the absence of the disturbance from source region 1 is predicted.

The test case consists of two baffled, simply supported, vibrating $5 \mathrm{~mm}$ steel panels, placed in the planes $z$ $= \pm 7.5 \mathrm{~cm}$. Both panels have dimensions $1 \times 1 \mathrm{~m}$ and a critical frequency of about $2.4 \mathrm{kHz}$, and both are driven by point forces of the same strength, but the driving points are not placed symmetrically. The two sources are modeled as modal sums, and the radiated sound field is calculated by a numerical approximation to Rayleigh's first integral. ${ }^{1}$ Reflections from the panels and baffles have been ignored. Ignoring such reflections for this configuration is evidently not realistic. However, no method can take account of such reflections, and the purpose of this test case is not to model a realistic situation but to compare the performance of the two methods with a realistically complicated sound field. Note that the measurement arrays are much smaller than the sources; this is no problem for the SONAH method. ${ }^{2,3,5,7}$

Figure 1 shows a comparison of the "true" and predicted undisturbed pressure and particle velocity in a diagonal across the primary prediction plane when the two panels are driven at $1.5 \mathrm{kHz}$. It is apparent that both methods give acceptable results in spite of the strong disturbance. The $p-u$ method predicts both the pressure and the particle velocity somewhat better than the DLA method.

Figure 2 shows the relative global errors of the predicted undisturbed pressure, particle velocity, and sound intensity for the same test case as functions of the frequency. The results are based on an ensemble of 20 pairs of vibrating panels with driving points at random positions. The relative global error is defined as the ratio of the Euclidian norm of the local deviations between predicted and true values to the Euclidean norm of the true quantity. ${ }^{3}$ As can be seen the relative errors of all quantities are of the order of $-17 \mathrm{~dB}$ (or $14 \%$ ) in the medium frequency range. On the whole the DLA method predicts the sound pressure somewhat better than the $p-u$ method, whereas the $p-u$ method predicts the particle velocity and the sound intensity somewhat better than the DLA method.

Finally, Fig. 3 compares the results of the $p-u$ and the DLA method with and without the disturbing sound field generated by a secondary source of the same strength as the primary source. Without the disturbing source the pressure is also predicted just from pressure measurements (in a single layer at $z=0$ ) and the particle velocity is predicted just from particle velocity measurements. It is clear that in the absence of disturbing sound from the other side of the measurement array the pressure is predicted better from the pressure in a single layer than with the $p-u$ or the DLA method, and the particle velocity is predicted better from the the particle velocity than with the $p-u$ or the DLA method. Under such circumstances the $p-u$ and the DLA methods give similar results. With the disturbing sound field the DLA method predicts the pressure somewhat better than the $p$ - $u$ method, whereas the $p-u$ method predicts the particle velocity slightly better than the DLA method.
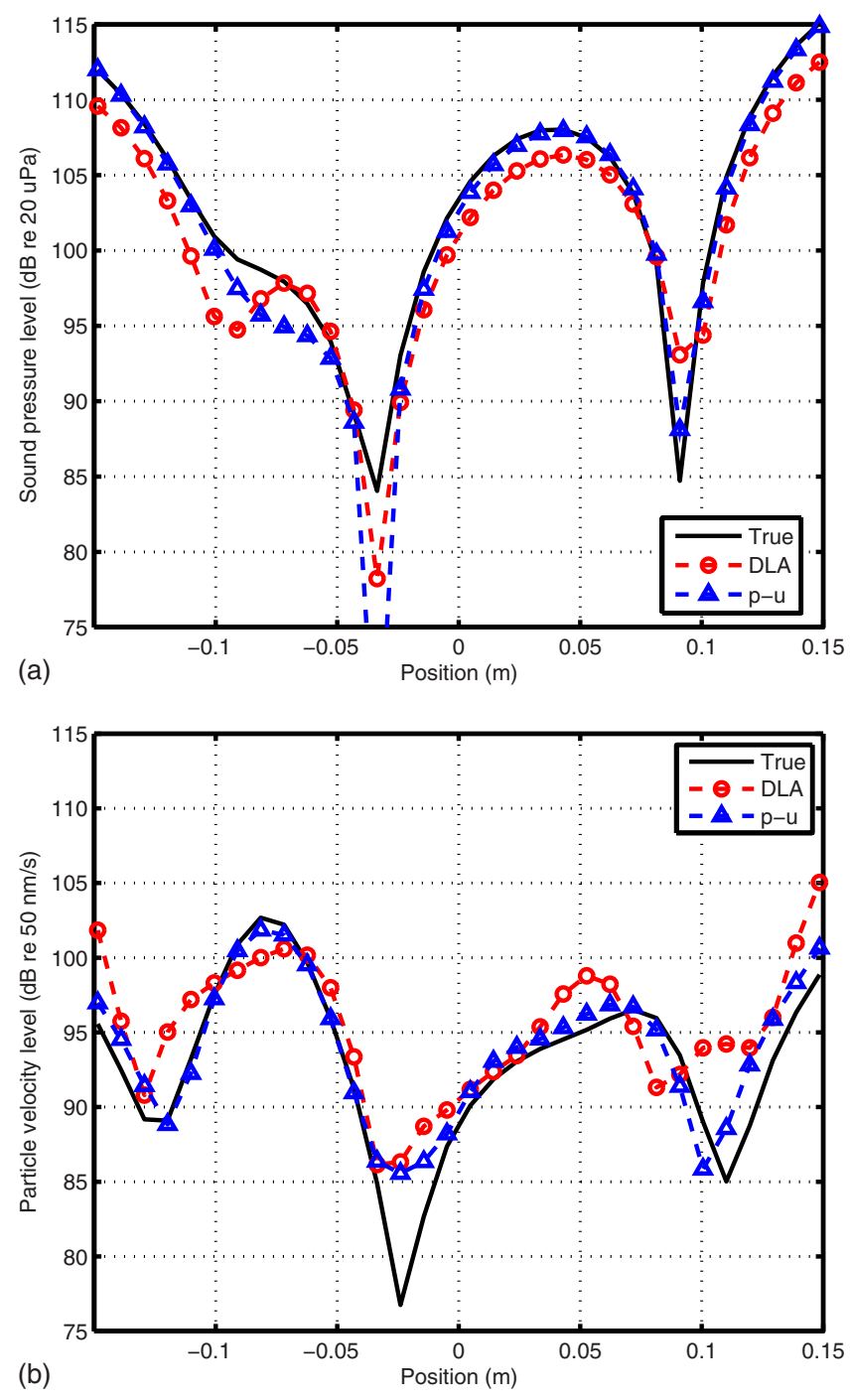

FIG. 1. (Color online) "True" and reconstructed undisturbed sound pressure (a) and particle velocity (b) in a diagonal across the primary prediction plane. Sources: Two baffled panels driven at $1.5 \mathrm{kHz}$ by point forces of the same strength.

\section{DISCUSSION}

In a previous investigation of SONAH it was concluded that prediction of the pressure from measurements of the pressure and prediction of the particle velocity from measurements of the particle velocity without disturbing sound from the other side of the measurement plane in general are better than predicting one quantity from the other. ${ }^{5}$ Thus it is perhaps not surprising that the $p-u$ method is no better than the DLA method in the absence of disturbing noise, and it is clear that these two methods should only be used when it is necessary to suppress noise coming from the other side of the measurement plane. In the presence of disturbing sound from the other side of the measurement plane, the $p$ - $u$ method relies on pressure- and particle velocity-based estimates of the same two quantities being identical; if this is not the case the disturbing sound will contaminate the prediction; this explains why the performance of the method is not significantly better than the performance of the DLA method. 


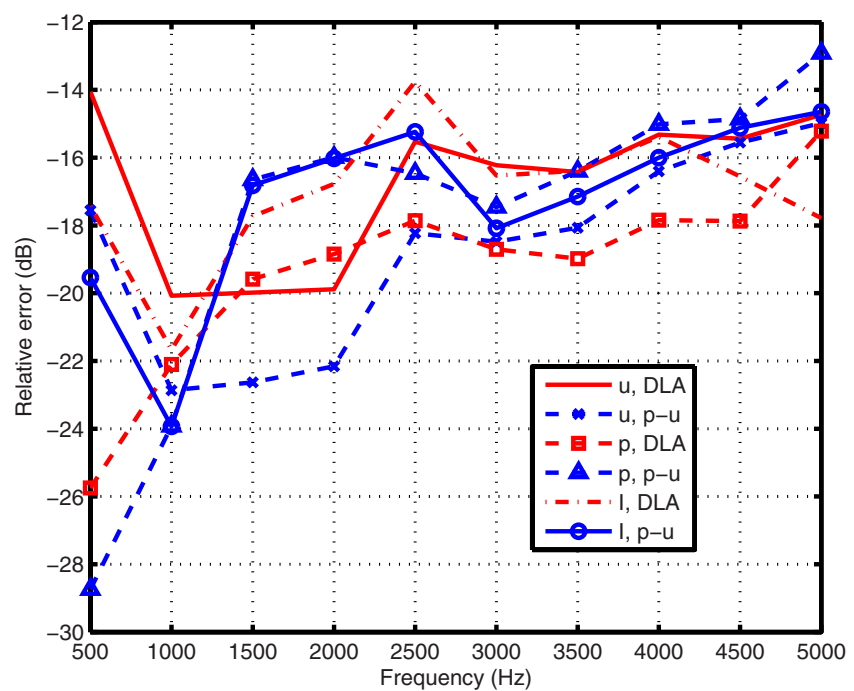

FIG. 2. (Color online) Relative global error of reconstructed undisturbed particle velocity, sound pressure, and sound intensity in the primary prediction plane as a function of the frequency. Sources: Two baffled panels driven by point forces of the same strength.

\section{CONCLUSIONS}

Statistically optimized near field acoustic holography can be based on measurements of the pressure in a plane, measurements of the normal component of the particle velocity in a plane, measurements of both quantities in the same plane, or measurements of the pressure in two parallel planes. Both the double layer method and the method based on measurements of pressure and particle velocity make it possible to distinguish between sound field components coming from the two sides of the measurement array and thus suppress even strong unwanted disturbing sound coming from the other side, and there is no significant difference between predictions determined with the two methods under such circumstances. However, in the absence of such disturbing sound field components then the most accurate prediction of the pressure near the source under test is obtained on the basis of pressure measurements in a single plane, and the most accurate prediction of the particle velocity is obtained from particle velocity measurements.

${ }^{1}$ E. G. Williams, Fourier Acoustics—Sound Radiation and Nearfield Acoustical Holography (Academic Press, San Diego, 1999).

${ }^{2}$ R. Steiner and J. Hald, "Near-field acoustical holography without the errors and limitations caused by the use of spatial DFT," Int. J. Acoust. Vib. 6, 83-89 (2001).

${ }^{3}$ J. Hald, "Patch near-field acoustical holography using a new statistically optimal method," Proceedings of Inter-Noise 2003, Jeju Island, Korea, 2003, pp. 2203-2210.

${ }^{4} \mathrm{~F}$. Jacobsen and Y. Liu, "Near field acoustic holography with particle velocity transducers," J. Acoust. Soc. Am. 118, 3139-3144 (2005).

${ }^{5}$ F. Jacobsen and V. Jaud, "Statistically optimized near field acoustic holog-
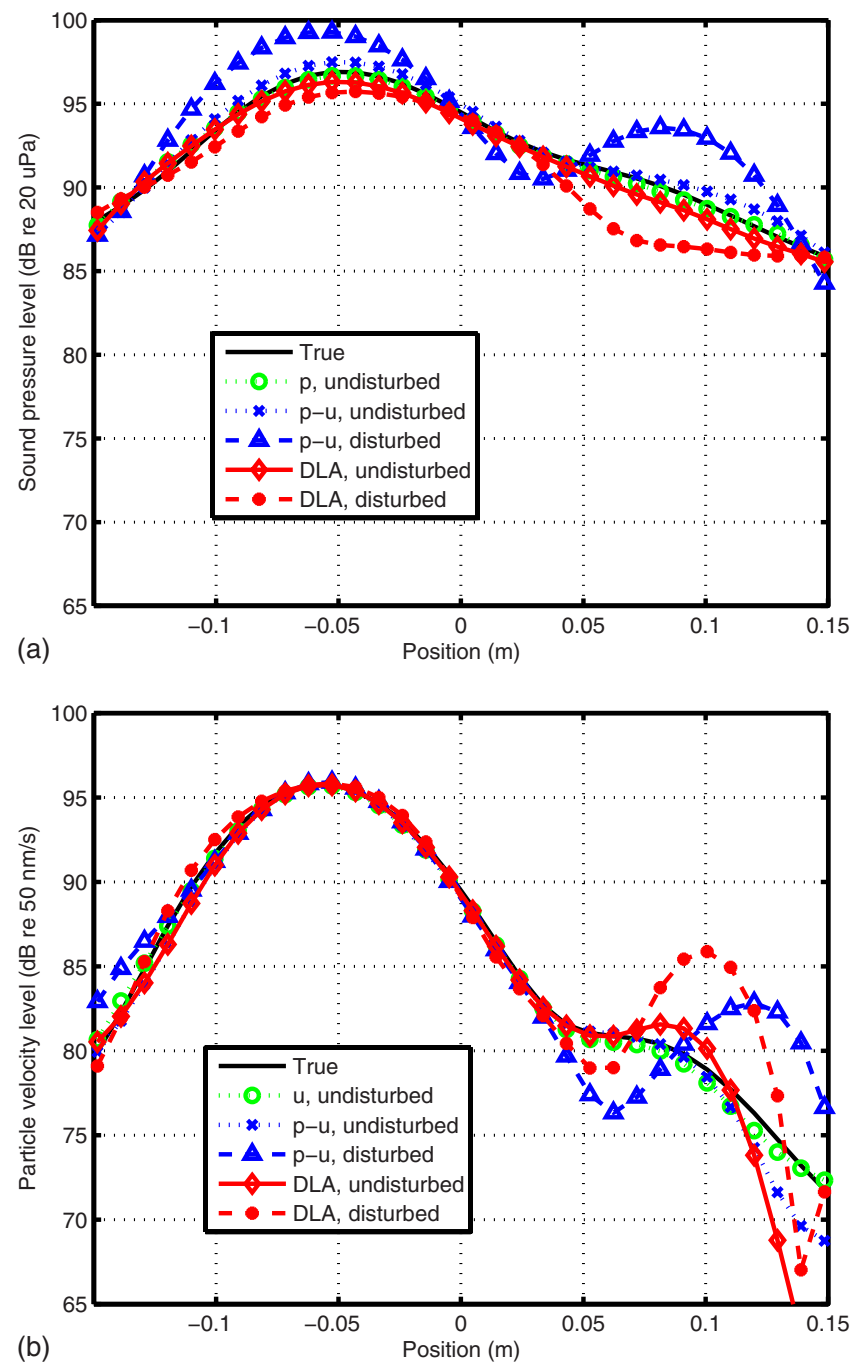

FIG. 3. (Color online) True and reconstructed undisturbed sound pressure (a) and particle velocity (b) in a diagonal across the primary prediction plane. Sources: Two baffled panels driven at $1.5 \mathrm{kHz}$ by point forces of the same strength. Results predicted without the disturbing secondary source are also shown.

raphy using an array of pressure-velocity probes," J. Acoust. Soc. Am. 121, 1550-1558 (2007).

${ }^{6} \mathrm{M}$. Tamura, "Spatial Fourier transform method of measuring transmission coefficients at oblique incidence. I. Theory and numerical examples," J. Acoust. Soc. Am. 88, 2259-2264 (1990).

${ }^{7}$ J. Hald, "Patch holography using a two-layer handheld array with an extended SONAH algorithm," Proceedings of Euronoise, Tampere, Finland, 2006.

${ }^{8} \mathrm{~F}$. Jacobsen and V. Jaud, "A note on the calibration of pressure-velocity sound intensity probes," J. Acoust. Soc. Am. 120, 830-837 (2006).

${ }^{9} \mathrm{~F}$. Jacobsen, X. Chen, and V. Jaud, "Weighted statistically optimised near field acoustic holography with pressure-velocity probes," Proceedings of 14th International Congress on Sound and Vibration, Cairns, Australia, 2007.

${ }^{10}$ P. C. Hansen, Rank-Deficient and Discrete Ill-Posed Problems: Numerical Aspects of Linear Inversion (SIAM, Philadelphia, 1997). 